this cohort, the 10-gene model had a predictive accuracy of $84 \%$, a sensitivity of $90 \%$, and a specificity of $80 \%$. In an independent cohort of 45 stage I lung adenocarcinomas, the 10-gene model predicted patient survival more accurately than did tumor stage (IA vs IB), tumor grade, age, or sex. Furthermore, patients with a good prognosis according to the 10-gene model had significantly different Kaplan-Meier survival curves to those with a poor profile $(P=0.008)$. The authors conclude that the 10-gene signature has prognostic value in lung adenocarcinoma.

Original article Bianchi F et al. (2007) Survival prediction of stage I lung adenocarcinomas by expression of 10 genes. J Clin Invest 117: 3436-3444

\section{Upgrading Gleason score 7 and tertiary grade 5 prostate cancer is warranted}

The Gleason score is a well-established indicator of prognostic significance in patients with prostate cancer, but there is still debate about how best to treat men with a Gleason score of $7(3+4$ or $4+3)$ and a tertiary pattern of 5 . The US International Society of Urologic Pathology has recommended that men with this Gleason score and pattern should have their cancer reclassified as Gleason score 8 or 9.

Patel et al. investigated the prognostic importance of Gleason score 7 with tertiary grade 5 compared with other Gleason scores in 2,370 men with clinical tumor category $1 \mathrm{c}-3 \mathrm{~b}$, nodenegative, and nonmetastatic prostate cancer. Gleason scores were assigned and Cox regression analysis was used to judge whether there was a significant association between Gleason score and the rate of disease progression.

Men with a Gleason score 7 and tertiary grade 5 experienced prostate-specific antigen (PSA) failure significantly more quickly than did men with Gleason score 7 without tertiary grade 5 (median time 5.0 years vs 6.7 years), and at a similar rate to men with Gleason score 8-10 (median time 5.1 years). Men with a Gleason score of 6 maintained low PSA levels for a significantly longer period (median time 15.4 years).

The authors conclude that it might be prudent to upgrade men with a Gleason score of 7 and a tertiary grade 5 to a higher level, and alter their treatment regimen accordingly.

Original article Patel AA et al. (2007) PSA failure following definitive treatment of prostate cancer having biopsy Gleason score 7 with tertiary Grade 5. JAMA 298: 1533-1538

\section{Combined therapy improves survival in advanced-stage endometrial cancer}

Early-stage endometrial cancer has an excellent prognosis; however, the survival rates for patients with advanced disease remain very low and an optimum management strategy has yet to be defined. A study by Secord et al. has evaluated the influence of combined adjuvant therapy on the survival of patients with advanced endometrial cancer.

This retrospective study included 356 patients with stage III or IV cancer, of whom $83 \%$ were optimally debulked and $17 \%$ received suboptimal cytoreduction. Postoperative radiotherapy alone and chemotherapy alone was administered to $48 \%$ and $29 \%$ of patients, respectively, while $23 \%$ of patients received combined chemotherapy and radiation. Overall survival (OS) and progression-free survival differed significantly between the three groups $(P<0.001)$. Patients who received chemotherapy alone had worse 3 -year OS and progression-free survival (33\% and $19 \%$, respectively) than did patients who received radiotherapy alone $(70 \%$ and $59 \%)$ or patients who received combined therapy $(79 \%$ and $62 \%)$. In comparison with the combinedtherapy group, the adjusted hazard ratio (HR) for OS was $2.01(P=0.012)$ for the radiotherapy group and $1.60(P=0.122)$ for the chemotherapy group. Optimally debulked patients who received either radiotherapy or chemotherapy alone had significantly higher risks for disease progression $(H R=1.80$ and $H R=1.84$, respectively; $P<0.05$ for both) and death $(\mathrm{HR}=2.64$; $P=0.004$ and $\mathrm{HR}=2.33 ; P=0.024)$ than did patients who received combined therapy.

The authors conclude that combined chemotherapy and radiation treatment increases survival in patients with advanced endometrial cancer compared with use of either modality alone.

Original article Secord AA et al. (2007) The role of multimodality adjuvant chemotherapy and radiation in women with advanced stage endometrial cancer. Gynecol Oncol 107: 285-291 